\title{
Tratamento ortopédico com aparelho de Herbst: ocorrem mudanças verticais no padrão de crescimento facial?
}

\author{
Luís Antônio de Arruda Aidar*, Gladys Cristina Dominguez**,

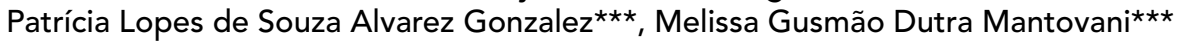

\begin{abstract}
Resumo
Objetivo: o objetivo deste estudo prospectivo de 32 adolescentes com má oclusão de Classe II, divisão 1, associada a retrognatismo mandibular, tratados com aparelho de Herbst, construído sobre bandas e coroas metálicas, foi avaliar cefalometricamente as possíveis mudanças no padrão de crescimento facial. Metodologia: as telerradiografias laterais foram obtidas ao início do tratamento (T1) e imediatamente após 12 meses de tratamento com o referido aparelho ortopédico (T2). Foram utilizados o quociente de Jarabak e o VERT de Ricketts (modificado) para determinação do padrão facial em T1 e T2. Resultados: utilizando o quociente de Jarabak, os resultados evidenciaram que 27 casos $(84,4 \%)$ apresentaram padrões hipodivergentes em Tl e permaneceram da mesma forma em T2. Cinco casos $(15,6 \%)$ apresentaram padrão neutro em T1 e não exibiram mudanças em T2. Quando avaliado o VERT de Ricketts (modificado), não ocorreram mudanças no padrão facial em 31 pacientes. Em apenas um caso ocorreu mudança do tipo facial. Conclusão: baseado nos resultados obtidos, pode-se concluir que, após 12 meses de tratamento com aparelho de Herbst, não ocorreram mudanças verticais no padrão de crescimento facial dos pacientes estudados.
\end{abstract}

Palavras-chave: Aparelho de Herbst. Cefalometria. Tipo facial.

\section{INTRODUÇÃO}

O aparelho de Herbst é um mecanismo telescópico bilateral ancorado nos arcos superior e inferior que mantém a mandíbula em posição postural anterior contínua durante todas as funções mandibulares ${ }^{14}$. Esse método de tratamento não depende da cooperação do paciente e, entre outras indicações, o aparelho era utilizado na correção de DTMs, tais como estalidos e bruxis$\mathrm{mo}^{14}$. Embora tenha sido proposto no início do século passado, existem poucas referências dessa técnica na literatura ortodôntica, até a mesma ter sido reintroduzida como método de tratamento, em $1979^{14}$.

De acordo com uma avaliação realizada em seis dos maiores laboratórios dos Estados Unidos,

\footnotetext{
* Professor doutor responsável pela disciplina de Ortodontia da Faculdade de Odontologia da Universidade Santa Cecília (Unisanta). Coordenador do curso de especialização em Ortodontia (Unisanta).

** Professora livre docente da disciplina de Ortodontia da Faculdade de Odontologia da Universidade de São Paulo (USP-SP).

*** Especialistas em Ortodontia pela Associação dos Cirurgiões-Dentistas de Santos, São Vicente e Região da Costa da Mata Atlântica (ACDSSV).
} 
entre os aparelhos ortopédicos funcionais, esse dispositivo mecânico funcional tem aumentado a sua popularidade ${ }^{12}$. No Brasil, sua utilização cresceu muito, principalmente após os cursos ministrados pelo Professor Hans Pancherz, da Alemanha.

Esse método de tratamento tem mostrado ser efetivo na correção da má oclusão de Classe II 1,2,13,14,21,24. Em adição ao possivel efeito de estímulo sobre o crescimento mandibular, a terapia com Herbst resulta em redirecionamento do crescimento da maxila, movimento mesial dos dentes inferiores e movimento distal dos dentes superiores $^{13,25}$. Todos esses fatores acabam fazendo parte dos mecanismos de correção da Classe II.

Tendo isso em mente, a atenção ao relacionamento vertical das bases ósseas e à maneira pela qual os diversos padrões de crescimento facial respondem à terapia com aparelhos funcionais é de suma importância para o sucesso do tratamento da má oclusão de Classe II ${ }^{15,21,23}$. Desta forma, torna-se de vital importância a avaliação dos efeitos verticais desse método de tratamento no padrão facial do paciente. A relação vertical entre a maxila e a mandíbula pode ser afetada pelos efeitos dentoalveolares do aparelho de Herbst, principalmente em pacientes que apresentam altura facial anterior aumentada, resultando em deterioração na estética facial ${ }^{15}$. Por outro lado, um estudo não revelou modificações significativas no padrão de crescimento vertical em pacientes com padrão de crescimento neutro e hipodivergente ${ }^{23}$.

Alguns trabalhos publicados ${ }^{11,23,24,25,29}$ descrevem diferentes tipos de sistemas de ancoragem. Dependendo do tipo facial do paciente ao início do tratamento, os clínicos devem estar atentos às diferentes mudanças dentofaciais induzidas no plano vertical pelos diferentes desenhos do aparelho de Herbst ${ }^{23}$.

O objetivo deste estudo foi avaliar cefalometricamente os possíveis efeitos verticais no padrão de crescimento facial em um grupo de adolescentes com retrognatismo mandibular tratados com aparelho ortopédico de Herbst.

\section{MATERIAL E MÉTODOS \\ Material}

Fizeram parte deste estudo 32 adolescentes, brasileiros, leucodermas, de ambos os gêneros (16 do gênero masculino e 16 do feminino) que foram tratados com o aparelho ortopédico de Herbst, utilizado para corrigir a má oclusão de Classe II, divisão 1, de Angle, associada a retrognatismo mandibular. A idade média pré-tratamento (T1) foi de 12 anos e 10 meses \pm 1 ano e 2 meses (variação de 10 anos e 11 meses a 15 anos e 10 meses).

Foram incluídos pacientes com as seguintes características: (1) aparência clínica de mandíbula retrognática, com ângulo ANB igual ou maior que $4^{\circ}$; (2) má oclusão de Classe II, divisão 1, dentadura permanente e ausência de mordida aberta anterior; (3) que estavam na fase do aparecimento do osso sesamoide ( $\mathrm{S}-3^{\circ}$ estágio de Björk e $\mathrm{Helm}^{7}$ ) até imediatamente depois de passado o pico máximo, havendo atingido o início do capeamento da falange medial do terceiro dedo $\left(\mathrm{FM}_{3}\right.$ cap $-4^{\circ}$ estágio de Björk e Helm7), na radiografia de mão e punho.

O comitê de Ética em Pesquisa da Universidade Federal de São Paulo/Hospital São Paulo analisou e aprovou o Projeto de Pesquisa em 12/06/2000 (Ref. CEP n ${ }^{\circ}$ 679/00).

Todos os pacientes foram tratados com o aparelho de Herbst modificado, com coroas de aço sobre os primeiros molares superiores e primeiros pré-molares inferiores, bandas ortodônticas sobre os primeiros pré-molares superiores e primeiros molares inferiores, um expansor Hyrax adaptado às coroas e bandas superiores e um arco lingual de Nance adaptado às coroas e bandas inferiores (Fig. 1). Apoios oclusais foram usados nos casos em que os segundos molares superiores e/ou inferiores estavam presentes. Foi necessária a expansão rápida da maxila em todos os pacientes em razão da deficiência transversal da maxila presente na má oclusão de Classe $\mathrm{II}^{1,2,27}$. A expansão rápida da maxila ocorreu, em média, nas duas primeiras semanas após a colocação do aparelho de Herbst. 

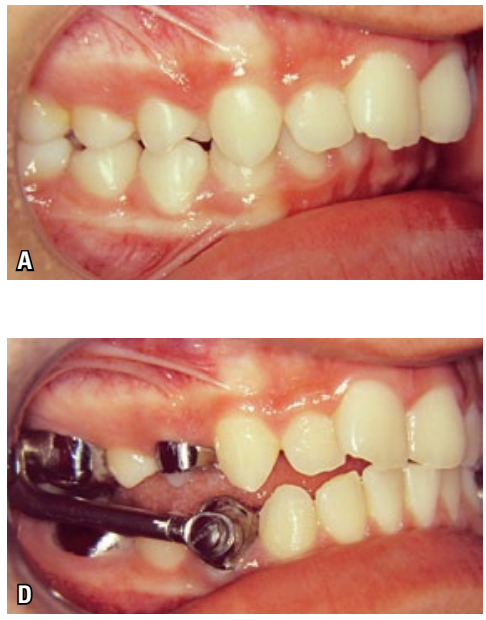
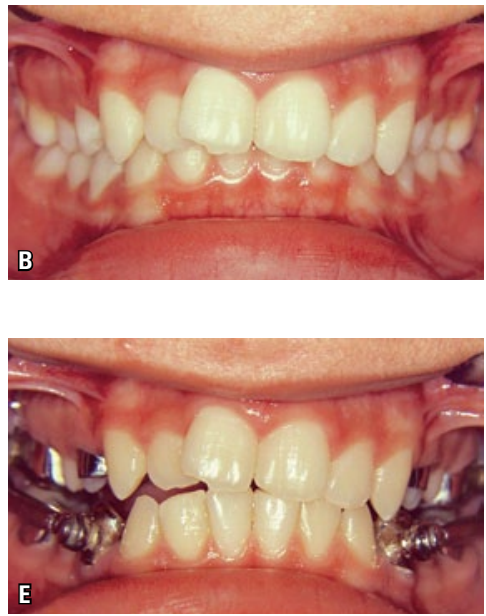
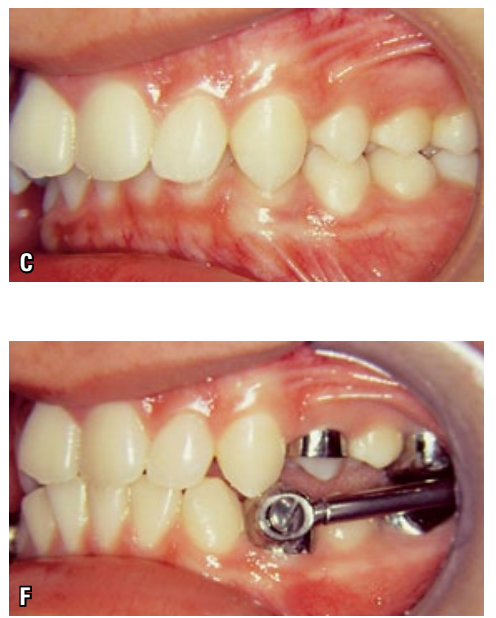
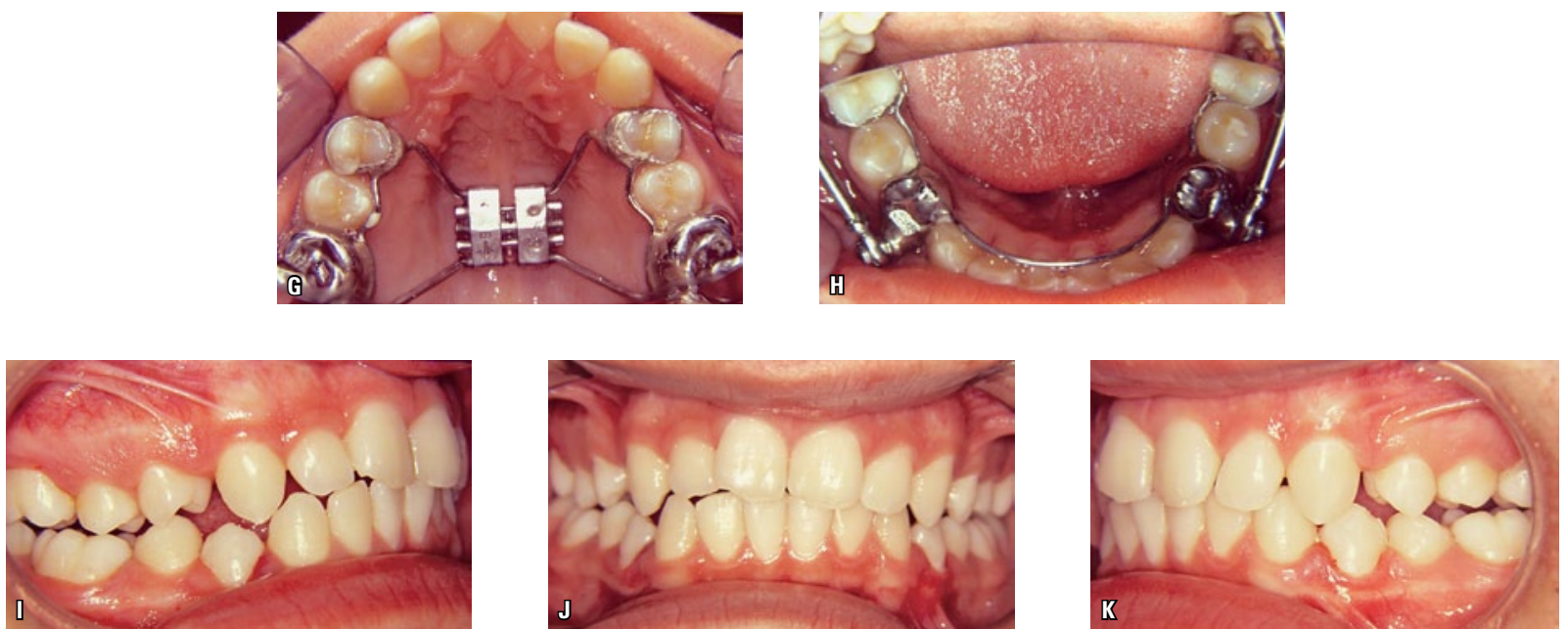

FIGURA 1 - Fotos intrabucais da sequência do tratamento com o aparelho de Herbst com bandas e coroas de aço.

Foram realizados avanços de até $6 \mathrm{~mm}$ ao início do tratamento. Quando necessário, avanços complementares foram realizados no terceiro mês. Avanços mandibulares assimétricos foram realizados em alguns casos, com objetivo de corrigir o desvio da linha média esquelética ${ }^{1,2}$.

Em todos os 32 pacientes, a terapia com aparelho de Herbst resultou em uma relação de Classe I ou Classe I sobrecorrigida dos arcos dentários.

\section{Métodos}

Os adolescentes foram avaliados por meio de telerradiografias laterais, imediatamente antes do início do tratamento (Tl) e após a terapia com o aparelho ortopédico de Herbst (T2), utilizado durante 12 meses para corrigir a má oclusão de Classe II, divisão 1, de Angle, associada a retrognatismo mandibular. As telerradiografias laterais foram realizadas sempre no mesmo cefalostato (marca B.F. Wehmer, EUA) e em um aparelho de raios $\mathrm{X}$ da marca $\mathrm{GE}^{\circledR}$ (General Eletrics, EUA), em norma lateral direita e em oclusão cêntrica. Nas telerradiografias, foram traçados cefalogramas em folhas de acetato, copiando os detalhes anatômicos de interesse para o traçado cefalométrico. Para medição das variáveis cefalométricas, foram utilizados transferidor e régua milimetrada com subdivisão de $0,5^{\circ}$ e $0,5 \mathrm{~mm}$, respectivamente. Foram avaliadas 
algumas variáveis cefalométricas da análise de Jarabak $^{26}$. Para avaliar o padrão de crescimento facial dos pacientes estudados, foi utilizado o quociente de Jarabak ${ }^{26}$ e o VERT de Ricketts ${ }^{4}$ (modificado).

\section{Variáveis cefalométricas da análise de Jarabak $^{26}$}

Foram utilizadas as seguintes variáveis cefalométricas lineares: S-N (base anterior do crânio, determinada pela união dos pontos sela e násio), S-Ar (base posterior do crânio, determinada pela união dos pontos sela e articular), Ar-Goc (plano do ramo da mandíbula, determinado pela união dos pontos articular e gônio construído), Me-Goc (plano mandibular, determinado pela união dos pontos mentoniano e gônio construído), S-Goc (altura facial posterior, determinada pela união dos pontos sela e gônio construído), N-Me (altura facial anterior, determinada pela união dos pontos násio e mentoniano), S-Gnc (longitude facial, determinada pela união dos pontos sela e gnátio construído) e N-Goc (profundidade facial, determinada pela união dos

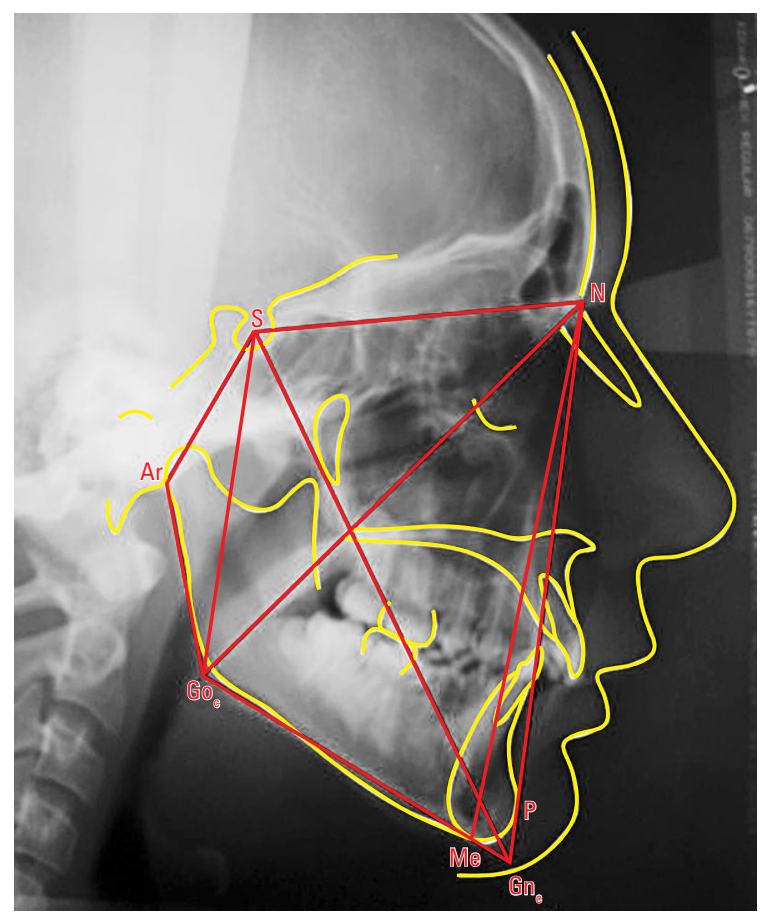

FIGURA 2 - Variáveis cefalométricas da análise de Jarabak. pontos násio e gônio construído) (Fig. 2).

\section{Quociente de Jarabak ${ }^{26}$ (FHR)}

Muitas análises são correntemente usadas para ajudar no diagnóstico da direção do crescimento. A morfologia facial tem sido caracterizada ${ }^{26}$ com base em três padrões distintos definidos pela Razão da Altura Facial (FHR) ou Quociente de Jarabak, isto é: a razão da Altura Facial Posterior (S-Goc) pela Altura Facial Anterior (N-Me), multiplicada por 100. Assim, obtém-se um percentual, o qual é representativo para descrever a morfologia facial (Fig. 3).

$$
(\mathrm{FHR})=(\mathrm{S}-\mathrm{Goc} / \mathrm{N}-\mathrm{Me}) \times 100
$$

Quando o percentual apresenta-se menor do que 59\%, é classificado como padrão de crescimento hiperdivergente; quando compreendido num intervalo entre $59 \%$ e $63 \%$, é definido como padrão de crescimento neutro; e acima de 63\%, como padrão de crescimento hipodivergente.

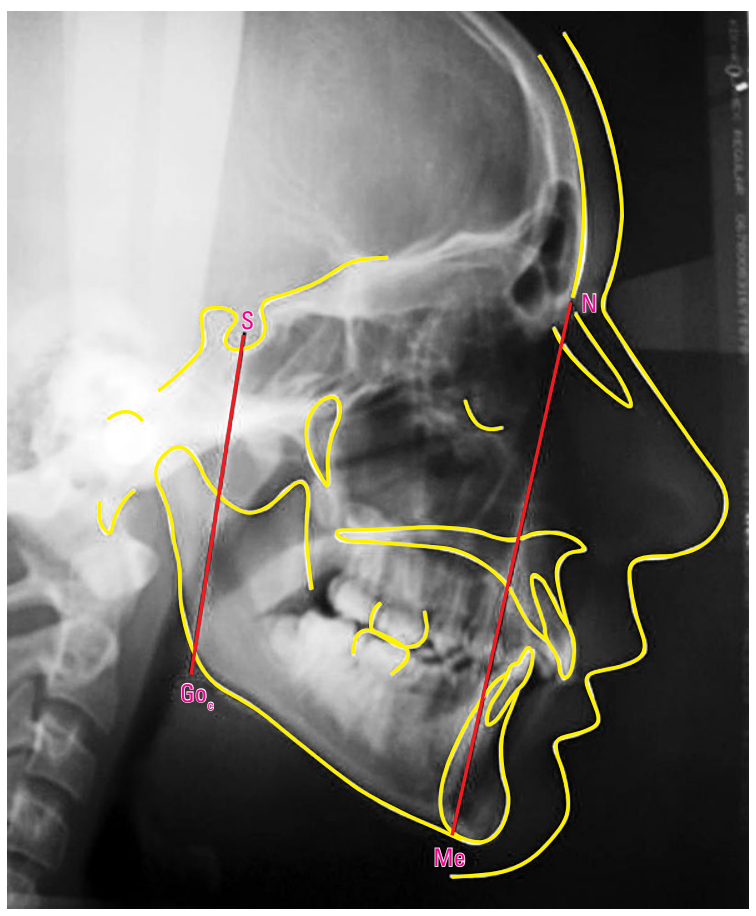

FIGURA 3 - Variáveis cefalométricas utilizadas no Quociente de Jarabak. 


\section{VERT de Ricketts ${ }^{19}$}

Empregando-se o método de Ricketts ${ }^{19}$, podem ser observados três tipos faciais: mesofacial, dolicofacial e braquifacial, dependendo se a direção de crescimento da face for para baixo ou para a frente, isto é, mais em direção vertical ou mais em direção horizontal, respectivamente. Neste trabalho foi usado o VERT de Ricketts ${ }^{4}$ (modificado), utilizando-se as estratificações para os tipos dolicofacial e braquifacial em suave, médio e severo. O tipo facial é definido a partir da determinação do VERT (quantidade de crescimento vertical), adotando-se as seguintes variáveis: ângulo do eixo facial - é o ângulo formado pelo plano Násio-Básio (N-Ba) com o Eixo Facial (PtGn); ângulo facial (profundidade) - é o ângulo formado pelo plano de Frankfurt (Po-Or) com o Plano Facial (N-P); ângulo do plano mandibular - ângulo formado pelo plano mandibular (Go$\mathrm{Me}$ ) e Plano de Frankfurt (Po-Or); altura da face inferior - ângulo formado pelas linhas Xi-ENA e $\mathrm{Xi}-\mathrm{Pm}$; arco mandibular - ângulo formado pelo Eixo Condilar (Xi-DC) e Eixo do Corpo (XiPm) (Fig. 4).

Após os cálculos para determinação do VERT, conforme descrito na literatura ${ }^{19,28}$, os pacientes foram classificados de acordo com o quadro 1.

\section{Método estatístico}

Testes, aplicados inicialmente, mostraram distribuição simétrica dos valores medidos. Por essa razão, o teste paramétrico foi aplicado para análise estatística.

Para avaliar possíveis diferenças entre as grandezas cefalométricas angulares e lineares estudadas ao início do tratamento (T1) e no final do tratamento (T2), foi aplicado o teste $t$ pareado. Os níveis de significância usados foram $p \leq 0,001$, $\mathrm{p} \leq 0,01$ e $\mathrm{p} \leq 0,05$.

\section{Erro do método}

Para avaliar a precisão do método utilizado, foram selecionadas, ao acaso, telerradiografias de

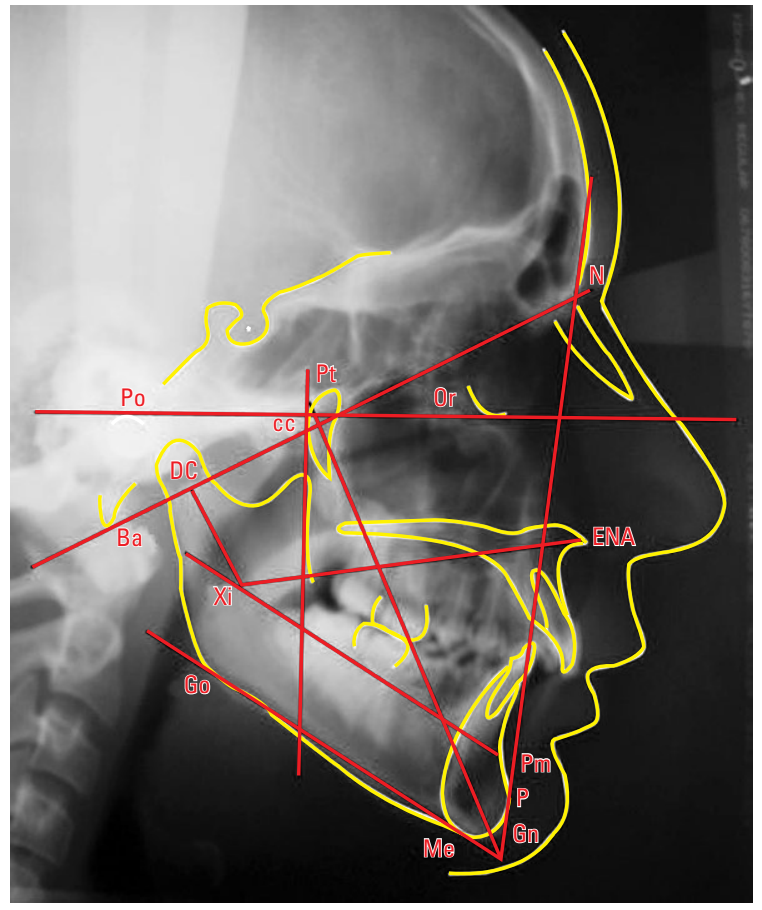

FIGURA 4 - Variáveis cefalométricas utilizadas no VERT de Ricketts ${ }^{4}$ (modificado).

\begin{tabular}{|cc|}
\hline TIPO FACIAL & VERT \\
\hline braquifacial severo & $+2,0$ \\
braquifacial médio & $+1,0$ \\
braquifacial suave & $+0,5$ \\
mesofacial & 0 \\
dolicofacial suave & $-0,5$ \\
dolicofacial médio & $-1,0$ \\
dolicofacial severo & $-2,0$
\end{tabular}

QUADRO 1 - Relação entre o VERT de Ricketts ${ }^{4}$ (modificado) e o tipo facial.

16 pacientes provenientes da amostra estudada (n = 32). Todas as telerradiografias foram traçadas e medidas novamente por um único operador após o período de um mês do traçado inicial, sendo aplicado o teste $t$ pareado para avaliação do erro sistemático. Com a diferença entre a primeira e a segunda mensuração de cada telerradiografia, aplicou-se a fórmula de Dahlberg para estimar o erro casual ${ }^{10}$. A fórmula aplicada foi $E=\sqrt{ } \sum \mathrm{d}^{2} / 2 n$, onde $\mathrm{d}$ é a diferença das medidas e $\mathrm{n}$ é o número dos casos retraçados da amostra. 


\section{RESULTADOS}

O erro sistemático não foi significativo em nenhum caso. O erro casual está apresentado nas tabelas 1 e 2.

Em T1, segundo os critérios de Siriwat e Jarabak $^{26}, 27$ pacientes apresentaram padrão de crescimento hipodivergente $(84,4 \%)$ e 5 neutro (15,6\%). Em T1, o VERT de Ricketts ${ }^{4}$ (modificado) apresentou a seguinte distribuição: 16 pacientes mesofaciais (50\%), 5 braquifaciais médio $(15,6 \%), 5$ dolicofaciais suave $(15,6 \%), 4$ braquifaciais suave $(12,5 \%), 1$ dolicofacial severo $(3,1 \%)$ e 1 braquifacial severo $(3,1 \%)$.

Em nenhum caso, de acordo com a avaliação de Siriwat e Jarabak ${ }^{26}$, ocorreram alterações dos padrões faciais de T1 para T2. Avaliando o VERT de Ricketts ${ }^{4}$ (modificado), em apenas um paciente o padrão passou de mesofacial, em T1, para braquifacial suave, em $\mathrm{T} 2$.

Todas as grandezas lineares da análise de Siriwat e Jarabak ${ }^{26}$ estudadas apresentaram diferenças estatísticas entre T1 e T2 (p < 0,001) (Tab. 1). As variáveis utilizadas para achar o VERT de Ricketts $^{4}$ (modificado) apresentaram os seguintes resultados entre T1 e T2: ângulo do eixo facial (n.s.); ângulo facial ( $\mathrm{p}<0,001)$; ângulo do plano mandibular (n.s.); altura da face inferior ( $<<0,001)$; arco mandibular (n.s.) (Tab. 2).

\section{DISCUSSÃO}

As possíveis alterações verticais decorrentes das correções no sentido anteroposterior das bases ósseas são motivo de preocupação no planejamento do tratamento ortodôntico, uma vez que os tipos faciais são imutáveis ${ }^{19}$. Isso significa que não há alterações durante toda a vida do paciente - trata-se de uma característica natural individual. Por outro lado, um estudo ${ }^{9}$ evidenciou uma tendência geral de rotação da face no sentido anti-horário entre a infância e a adolescência nos três tipos faciais. Esses tipos faciais, entretanto, alteram-se facilmente, tornando-se mais verticais

TABELA 1 - Média, desvio-padrão (d.p.) das variáveis cefalométricas lineares (mm) em T1 e T2 (comparação das avaliações quantitativas com testes $\mathrm{t}$ - pareados) e erro casual (T1 e T2).

\begin{tabular}{|c|c|c|c|c|c|c|}
\hline & & \multirow{2}{*}{$\mathbf{T 1}$} & \multirow{2}{*}{ T2 } & \multirow{2}{*}{$\begin{array}{l}\text { SIENIFICÂNCIA } \\
\text { (P) }\end{array}$} & \multicolumn{2}{|c|}{ FÓRMULA DE DAHLBERG ERRO CASUAL } \\
\hline & & & & & $\mathrm{T} 1$ & T2 \\
\hline \multirow{2}{*}{ S-N } & média & 72,42 & 73,72 & \multirow{2}{*}{$<0,001 * * *$} & \multirow{2}{*}{0,45} & \multirow{2}{*}{0,34} \\
\hline & d. p. & 3,75 & 3,91 & & & \\
\hline \multirow{2}{*}{ S-Ar } & média & 36,13 & 37,27 & \multirow{2}{*}{$<0,001 * * *$} & \multirow{2}{*}{0,55} & \multirow{2}{*}{0,48} \\
\hline & d. p. & 3,37 & 3,56 & & & \\
\hline \multirow{2}{*}{ Ar-Goc } & média & 45,31 & 47,28 & \multirow{2}{*}{$<0,001 * * *$} & \multirow{2}{*}{0,52} & \multirow{2}{*}{0,41} \\
\hline & d. p. & 4,13 & 4,18 & & & \\
\hline \multirow{2}{*}{ Me-Goc } & média & 70,14 & 72,86 & \multirow{2}{*}{$<0,001 * * *$} & \multirow{2}{*}{0,61} & \multirow{2}{*}{0,41} \\
\hline & d. p. & 4,12 & 3,91 & & & \\
\hline \multirow{2}{*}{ S-Goc } & média & 77,80 & 80,52 & \multirow{2}{*}{$<0,001^{* * *}$} & \multirow{2}{*}{0,54} & \multirow{2}{*}{0,43} \\
\hline & d. p. & 5,12 & 5,66 & & & \\
\hline \multirow{2}{*}{$\mathrm{N}-\mathrm{Me}$} & média & 118,36 & 121,61 & \multirow{2}{*}{$<0,001 * * *$} & \multirow{2}{*}{0,40} & \multirow{2}{*}{0,41} \\
\hline & d. p. & 6,06 & 6,31 & & & \\
\hline \multirow{2}{*}{ S-Gnc } & média & 126,56 & 131,38 & \multirow{2}{*}{$<0,001 * * *$} & \multirow{2}{*}{0,45} & \multirow{2}{*}{0,52} \\
\hline & d. p. & 6,19 & 6,45 & & & \\
\hline \multirow{2}{*}{ N-Goc } & média & 119,89 & 122,38 & \multirow{2}{*}{$<0,001 * * *$} & \multirow{2}{*}{0,33} & \multirow{2}{*}{0,39} \\
\hline & d. p. & 6,47 & 6,82 & & & \\
\hline
\end{tabular}

\footnotetext{
*** estatisticamente significativo ao nível de 0,1\%.
} 
TABELA 2 - Média, desvio-padrão (d.p.) das variáveis cefalométricas angulares (graus) em T1 e T2 (comparação das avaliações quantitativas com testes $\mathrm{t}$ - pareados) e erro casual (T1 e T2).

\begin{tabular}{|c|c|c|c|c|c|c|}
\hline & & \multirow{2}{*}{$\mathrm{T1}$} & \multirow{2}{*}{ T2 } & \multirow{2}{*}{$\begin{array}{l}\text { SIGNIFICÂNCIA } \\
\text { (P) }\end{array}$} & \multicolumn{2}{|c|}{ FÓRMULA DE DAHLBERG ERRO CASUAL } \\
\hline & & & & & $\mathrm{T} 1$ & T2 \\
\hline \multirow{2}{*}{ ângulo do eixo facial } & média & 89,27 & 89,41 & \multirow{2}{*}{0,534 n.s. } & \multirow{2}{*}{0,47} & \multirow{2}{*}{0,56} \\
\hline & d. p. & 3,39 & 3,67 & & & \\
\hline \multirow{2}{*}{$\begin{array}{c}\text { ângulo facial } \\
\text { (profundidade) }\end{array}$} & média & 88,16 & 89,11 & \multirow{2}{*}{$* * *$} & \multirow{2}{*}{0,61} & \multirow{2}{*}{0,53} \\
\hline & d. p. & 3,13 & 3,49 & & & \\
\hline \multirow{2}{*}{$\begin{array}{l}\text { ângulo do plano } \\
\text { mandibular }\end{array}$} & média & 23,69 & 23,67 & \multirow{2}{*}{0,909 n.s. } & \multirow{2}{*}{0,64} & \multirow{2}{*}{0,75} \\
\hline & d. p. & 4,24 & 4,38 & & & \\
\hline \multirow{2}{*}{ altura da face interior } & média & 46,23 & 46,88 & \multirow{2}{*}{$* * *$} & \multirow{2}{*}{0,43} & \multirow{2}{*}{0,53} \\
\hline & d. p. & 3,99 & 4,08 & & & \\
\hline \multirow{2}{*}{ arco mandibular } & média & 32,66 & 32,66 & \multirow{2}{*}{1,000 n.s. } & \multirow{2}{*}{0,61} & \multirow{2}{*}{0,51} \\
\hline & d. p. & 4,01 & 3,88 & & & \\
\hline
\end{tabular}

n.s. = não-significativo.

*** estatisticamente significativo ao nível de 0,1\%.

se não forem tomados certos cuidados no planejamento do tratamento biomecânico. Dessa forma, acredita-se que a manutenção do tipo facial do paciente seja um fator de estabilidade pós-tratamento, pois o equilíbrio neuromuscular, nessas circunstâncias, ficará preservado ${ }^{28}$.

No presente trabalho, 27 dos 32 pacientes $(84,4 \%)$ apresentaram predominantemente padrões com tendência para crescimento no sentido horizontal ${ }^{26}$. O VERT de Ricketts ${ }^{4}$ (modificado) mostrou que 26 dos 32 pacientes $(81,2 \%)$ apresentaram predominantemente padrões equilibrados (50\%) ou com tendência para crescimento no sentido horizontal $(31,2 \%)$. Seis pacientes da amostra $(18,7 \%)$ apresentaram tendência vertical de crescimento. Não foi encontrada a mesma leitura para todos os pacientes, entre os dois métodos utilizados para determinação dos tipos faciais, corroborando os resultados de outro estudo prévio ${ }^{22}$. Na realidade, os dois métodos apresentam abordagens diferentes e complementares. Essa maior prevalência de padrões hipodivergentes, encontrada na presente amostra, não está em concordância com a investigação ${ }^{3}$ que encontrou uma maior prevalência de padrões de crescimento facial neutro em um grupo portador de má oclusão de Classe II, divisão 1. Também não confirmou os resultados de uma pesquisa ${ }^{8}$ que avaliou, em São Paulo, as telerradiografias em norma lateral de um grupo de 157 adolescentes consecutivos, com má oclusão de Classe II, divisão 1, e retrognatismo mandibular, idade média de 11 anos e 3 meses ( \pm 1 ano e 5 meses) e ângulo ANB igual ou maior que $4^{\circ}$. Os autores ${ }^{8}$ verificaram que, pelo VERT de Ricketts ${ }^{4}$, o maior número de adolescentes apresentava um tipo dolicofacial (48\%), seguido do tipo mesofacial (33\%) e com menor frequência o braquifacial (19\%). Essa diferença deve-se ao fato de que, na presente pesquisa, ao selecionar os pacientes, foi levado em consideração o prognóstico para a estabilidade a longo prazo, que parece ser dependente de um padrão de crescimento favorável pós-tratamento ${ }^{16}$. Dessa forma, os pacientes com mordida aberta anterior, mais frequente em padrões verticais ${ }^{5}$, foram excluídos da amostra.

As alterações sagitais, verticais e transversais, avaliadas clinicamente, que ocorreram nos pacientes da presente pesquisa, nos primeiros meses de tratamento utilizando o aparelho de Herbst, foram bastante evidentes. Quando se instala o aparelho de Herbst sobre bandas e coroas, a projeção mandibular, que corresponde à posição terapêutica imposta pela mordida construtiva, cria uma desoclusão na 
região posterior. A referida desoclusão é compensada durante os primeiros meses de tratamento, em decorrência do crescimento vertical alveolar, que se expressa pela ausência de contatos oclusais verticais. Nesse momento, fazem-se necessários apoios oclusais nos segundos molares, para evitar a extrusão diferenciada desses dentes. No presente estudo, observou-se que, embora tenham acontecido essas mudanças adaptativas de crescimento dentoalveolar, elas não influenciaram de forma desfavorável o tipo facial de $\mathrm{T} 1$ para $\mathrm{T} 2$.

Outro aspecto a considerar é a mordida cruzada que se instalou no momento da colocação do aparelho de Herbst. Grande parte das más oclusões de Classe II, associadas à retrusão mandibular, apresentaram deficiências transversais na maxila, evidenciadas com o avanço sagital promovido pelo aparelho de Herbst. Além disso, a expansão maxilar reduziu interferências oclusais e mudanças funcionais pela acomodação na porção mais larga do arco inferior posicionado anteriormente.

Em média, uma semana após a instalação dos aparelhos, o expansor Hyrax começou a ser ativado, com consequente abertura da sutura palatina mediana, em um protocolo preestabelecido de uma volta completa no primeiro dia e meia volta nos dias subsequentes, até que houvesse sobrecorreção. Durante essa fase, ocorreu abertura da mordida, avaliada clinicamente, algumas vezes diminuindo o efeito da mordida construtiva inicial.

Com respeito à época do tratamento, os pacientes dessa pesquisa iniciaram-no quando estavam no surto de crescimento puberal, avaliado na radiografia de mão e punho. Todavia, os clínicos devem lembrar que existem grandes variações individuais nas respostas esqueléticas e dentárias com esse método de tratamento ${ }^{17}$.

No presente estudo, em nenhum caso, segundo os critérios de Siriwat e Jarabak ${ }^{26}$, ocorreram alterações dos padrões faciais de $\mathrm{T} 1$ para $\mathrm{T} 2$. Foram encontrados na literatura estudos que utilizaram diferentes metodologias com relação ao tempo de tratamento e tipos de aparelho de Herbst, ficando, dessa forma, difícil a comparação com os resultados aqui encontrados. Pode ser citada uma pesquisa com o aparelho de Herbst utilizado em pacientes normohipodivergentes, onde os autores ${ }^{23}$ não encontraram mudanças significativas no padrão de crescimento vertical, concordando com os resultados do presente estudo. Durante um tempo de observação também de 12 meses, outra investigação ${ }^{24}$, que utilizou a versão de aparelho de Herbst sobre splints acrílicos, apresentou concordância com os presentes resultados. Importante notar que esse estudo poderia ter apresentado resultados diferentes, já que esse modelo, por recobrir as superfícies oclusais, não libera o crescimento dentoalveolar durante a fase ativa do tratamento com Herbst. Outros estudos ${ }^{15,20,25}$, utilizando diferentes desenhos do aparelho de Herbst, também não mostraram mudanças no ângulo do plano mandibular. Avaliando o VERT de Ricketts ${ }^{4}$ (modificado), em apenas 1 paciente $(3,1 \%)$ ocorreu alteração de $\mathrm{T} 1$ para $\mathrm{T} 2$ para um padrão mais horizontal. $\mathrm{O}$ caso $\mathrm{n}^{\circ} 8$ teve um aumento dos ângulos do eixo facial, profundidade facial e arco mandibular, passando de mesofacial, em Tl, para braquifacial suave, em T2, corroborando os resultados de um estudo prévio $^{23}$. Entretanto, nessa investigação os autores utilizaram o aparelho de Herbst associado a um splint de acrílico e à ancoragem extrabucal de tração alta. Na presente amostra - embora com poucos pacientes apresentando padrão de crescimento vertical em T1, de acordo com o VERT de Ricketts $^{4}$ (modificado) (6 casos $=18,75 \%$ ), e alguns deles com sobremordida e excessiva altura facial -, não foram encontrados efeitos deletérios do tratamento nos pacientes. $\mathrm{O}$ aumento na altura facial anterior ocorre com o crescimento para baixo do plano mandibular de forma paralela ${ }^{15}$. O tratamento com aparelho de Herbst tem mostrado um aumento do crescimento condilar na direção sagital terapêutica desejada $^{21}$, coincidindo com a direção de crescimento condilar em indivíduos hiperdivergentes ${ }^{6}$, sem resultar em rotação para baixo e para trás da mandíbula ${ }^{20}$. Dessa forma, indivíduos com ângulo do plano mandibular alto têm bom prognóstico na 
terapia com Herbst. Foram encontrados, nos resultados desse estudo, em média, aumentos significativos $(\mathrm{p}<0,001)$ da altura facial anterior $(\mathrm{N}-\mathrm{Me})$, altura da face inferior (Xi-ENA.Xi-Pm) e altura facial posterior (S-Goc) (Tab. 1, 2) não alterando o padrão facial do paciente, segundo os critérios de Siriwat e Jarabak $^{26}$, e o VERT de Ricketts ${ }^{4}$ (modificado). Isso parece lógico, especialmente porque o aumento na altura facial anterior durante o Herbst é devido ao efeito geométrico do reposicionamento mandibular anterior e ao aumento do comprimento mandibu$\operatorname{lar}^{20}$ ( $\mathrm{p}<0,001$ - Goc-Me) (Tab. 1).

Alguns estudos têm mostrado que o desenvolvimento vertical do ramo aumenta durante o tratamento com ativador ${ }^{30} \mathrm{e} \mathrm{Herbst}^{18}$. É importante ressaltar que, na presente pesquisa, o crescimento da base posterior do crânio (S-Ar) associado ao crescimento do ramo mandibular (Ar-Goc) aumentou significativamente $(p<0,001)$ entre T1 e T2, contribuindo para o aumento da altura facial posterior. As grandezas S-Gnc e N-Goc também tiveram modificações significativas $(\mathrm{p}<0,001)$ em razão do aumento das alturas faciais anterior e posterior. Pelo fato de não ter sido utilizado um grupo controle, em razão da idade oportuna que os pacientes apresentavam no momento do tratamento, fica difícil saber quais foram os efeitos do tratamento e do crescimento. $\mathrm{O}$ tratamento com Herbst seria mais útil naqueles casos de Classe II com falta de desenvolvimento vertical na altura facial anterior ${ }^{15}$. Por outro lado, resultados de um estudo ${ }^{21}$ mostraram que as mudanças esqueléticas e dentárias que contribuíram para a correção da Classe II não dependeram da relação vertical das bases ósseas.

Embora não tenham ocorrido alterações verticais significativas com a correção da Classe II dos casos tratados, é de suma importância o acompanhamento longitudinal desse grupo de adolescentes para avaliação da estabilidade dos resultados obtidos.

Finalmente, todos os pacientes desse trabalho foram submetidos a uma segunda fase do tratamento ortodôntico, com montagem de aparelhagem fixa superior e inferior, com o objetivo de refinamento da oclusão. Após essa fase, novas avaliações serão realizadas no intuito de verificar a estabilidade do padrão facial dos pacientes estudados do início ao final do tratamento ortodôntico.

\section{CONCLUSÃO}

Baseado nos resultados obtidos, pode-se concluir que, após 12 meses de tratamento com aparelho de Herbst, não ocorreram mudanças verticais que alterassem os padrões de crescimento facial dos pacientes estudados.

Enviado em: fevereiro de 2007 Revisado e aceito: junho de 2008

\title{
Orthopedic treatment with the Herbst appliance: Do vertical changes occur in the facial growth pattern?
}

\begin{abstract}
Objective: The aim of this prospective study was to evaluate through the exam of cephalograms the occurrence of changes in facial growth pattern. The sample was composed by 32 adolescent with Angle Class II, division 1 malocclusion and mandibular retrognathism. All patients were treated with the Herbst appliance built over bands and metal crowns. Methodology: Lateral cephalograms were taken at the beginning of treatment (T1) and immediately after 12 months of treatment with Herbst appliance (T2). Jarabak's coefficient and Ricketts' VERT (modified) were used to establish the facial pattern in T1 and T2. Results: According to Jarabak's coefficient, in T1, 84.4\% (27 cases) presented hypodiverging pattern and $15.6 \%$ (5 cases) presented neutral pattern. The facial growth pattern was maintained in all patients from T1 to T2. When the (modified) Ricketts' VERT was used, 31 patients showed the same growth pattern from T1 to T2. Only one patient exhibited a different pattern. Conclusion: Based on the results it can be concluded that, after 12 months of treatment with the Herbst appliance, there were no vertical changes in the facial growth pattern of the studied subjects.
\end{abstract}

Keywords: Herbst appliance. Cephalometrics. Facial pattern. 


\section{REFERÊNCIAS}

1. AIDAR, L. A. A. Posição do côndilo e disco das articulações temporomandibulares à ressonância magnética em adolescentes com retrognatismo mandibular tratados com aparelho de Herbst. 2003. Tese (Doutorado)-Escola Paulista de Medicina, Universidade Federal de São Paulo, São Paulo, 2003.

2. AIDAR, L. A. A.; ABRAHÃO, M.; YAMASHITA, H. K.; DOMINGUEZ, G. C. Herbst appliance therapy and temporomandibular joint disc position: A prospective longitudinal magnetic resonance imaging study. Am. J. Orthod. Dentofacial Orthop., St. Louis, v. 129, no. 4, p. 486-496, Apr. 2006

3. AIDAR, L. A. A.; SCANAVINI, M. A. Estudo comparativo cefalométrico radiográfico dos padrões de crescimento facial em pacientes portadores de oclusão normal e maloclusões de Classe I; Classe II, divisão 1; Classe II, divisão 2 e Classe III de Angle de acordo com Siriwat \& Jarabak. Ortodontia, São Paulo, v. 22, n. 2, p. 31-52, maio/ago. 1989.

4. ALBUQUERQUE, C. M.; VIGORITO, J. W. Estudo cefalométrico radiográfico empregando a análise de Ricketts na avaliação dos padrões dentofaciais de pacientes portadores de maloclusão de Classe II, divisão 1 , tratados durante a fase de dentição mista. Ortodontia, São Paulo, v. 23, n. 2, p. 11-28, 1990.

5. ALMEIDA, R. R.; URSI, W. J. S. Anterior open bite: Etiology and treatment. Oral Health, Don Mills, v. 80, no. 1, p. 27-31, Jan. 1990

6. BJÖRK, A. Prediction of mandibular growth rotation. Am. J. Orthod., St. Louis, v. 55, no. 6, p. 585-599, Jun. 1969

7. BJÖRK, A.; HELM, S. Prediction of the age of maximum pubertal growth in body height. Angle Orthod., Appleton, v. 37 , no. 2 , p. 134-143, 1967.

8. CARVALHO, P. L.; DOMINGUEZ-RODRIGUEZ, G. C. Estudo cefalométrico radiográfico da correlação entre o tipo facial e as variações da inclinação do plano oclusal em adolescentes com maloclusão de Classe II, divisão $1^{\text {a }}$ e retrognatismo mandibular. Ortodontia, São Paulo, v. 36, n. 3, p. 16-26, 2003.

9. COTRIM-FERREIRA, F. A.; PANELLA, J.; FERREIRA, F. V.; SCAVONE JÚNIOR, H.; MARTINS, D. R.; LIBERTI, E. A. Os tipos faciais e suas correlações com a base do crânio: estudo cefalométrico longitudinal. Ortodontia, São Paulo, v. 34 n. 3, p. 8-17, set./dez. 2001

10. HOUSTON, W. J. B. The analysis of errors in orthodontic measurements. Am. J. Orthod., St. Louis, v. 83, no. 5, p. 382-390, 1983

11. McNAMARA, J. A. Clinical management of the acrylic splint Herbst appliance. Am. J. Orthod., St. Louis, v. 94, no. 2, p. 142-149, Aug. 1988.

12. PANCHERZ, H. History, background, and development of the Herbst appliance. Semin. Orthod., Philadelphia, v. 9, no. 1, p. 3-11, 2003.

13. PANCHERZ, $H$. The mechanism of Class II correction in Herbst appliance treatment: A cephalometric investigation. Am. J. Orthod., St. Louis, v. 82, no. 2, p. 104-113, 1982.

14. PANCHERZ, H. Treatment of Class II malocclusions by bite jumping with the Herbst appliance: A cephalometric investigation. Am. J. Orthod., St. Louis, v. 76, no. 4, p. 423-441, 1979.

15. PANCHERZ, H. Vertical dentofacial changes during Herbst appliance treatment: A cephalometric investigation. Swed. Dent. J., Jönköping, v. 15, p. 189-196, 1982. Supplement.
16. PANCHERZ, H.; FACKEL, U. The skeletofacial growth pattern pre- and post-dentofacial orthopaedics: A long-term study of Class II malocclusions treated with the Herbst appliance. Eur. J. Orthod., Oxford, v. 12, no. 2, p. 209-218, 1990.

17. PANCHERZ, H.; HÄGG, U. Dentofacial orthopedics in relation to somatic maturation: An analysis of 70 consecutive cases treated with the Herbst appliance. Am. J. Orthod., St. Louis, v. 88 , no. 4 , p. $273-287,1985$.

18. PANCHERZ, H.; LITTMANN, C. Morphologie und Lage des Unterkiefers bei der Herbst-Behandlung. Eine kephalometrische Analyse der Veränderungen bis zum Wachstumsabschlub. Inf. Orthod. Kieferorthop., München, v. 21, p. $493-513,1989$.

19. RICKETTS, R. M. Orthodontic diagnosis and planning. Philadelphia: Saunders, 1982. v. 1, p. 107-125.

20. RUF, S.; PANCHERZ, H. The effect of Herbst appliance treatment on the mandibular plane angle: A cephalometric roentgenographic study. Am. J. Orthod. Dentofacial Orthop., St. Louis, v. 110 , no. 2, p. 225-230, 1996.

21. RUF, S.; PANCHERZ, H. The mechanism of Class II correction during Herbst therapy in relation to the vertical jaw base relationship: A cephalometric roentgenographic study. Angle Orthod., Appleton, v. 67, no. 4, p. 271-276, 1997.

22. SCANAVINI, C.; VIGORITO, J. W. Estudo cefalométricoradiográfico das possíveis correlações existentes entre as análises de Vigorito, Ricketts e Siriwat \& Jarabak na definição dos tipos faciais, em indivíduos leucodermas. Ortodontia, São Paulo, v. 34, n. 3, p. 27-41, 2001

23. SCHIAVONI, R.; GRENGA, V.; MACRI, V. Treatment of Class II high angle malocclusions with the Herbst appliance: A cephalometric investigation. Am. J. Orthod. Dentofacial Orthop., St. Louis, v. 102, no. 5, p. 393-409, 1992.

24. SCHÜTZ, T. C. B.; VIGORITO, J. W.; DOMINGUEZRODRÍGUEZ, G. C. Avaliação cefalométrica-radiográfica das modificações esqueléticas e do perfil facial decorrentes do tratamento com o aparelho de Herbst em adolescentes com maloclusão de Classe II, divisão $1^{\text {a }}$ de Angle. Parte II. Ortodontia, São Paulo, v. 36, n. 1, p. 44-61, 2003.

25. SIDHU, M. S.; KHARBANDA, O. P.; SIDHU, S. S. Cephalometric analysis of changes produced by a modified Herbst appliance in the treatment of Class II division 1 malocclusion. Br. J. Orthod., Oxford, v. 22, no. 1, p. 1-12, 1995.

26. SIRIWAT, P. P.; JARABAK, J. R. Malocclusion and facial morphology is there a relationship? Angle Orthod., Appleton, v. 55 , no. 2 , p. 127-138, 1985

27. TOLLARO, I.; BACCETTI, T.; FRANCHI, L.; TANASESCU, C. D. Role of posterior transverse interarch discrepancy in Class II, division 1 malocclusion during the mixed dentition phase. Am. J. Orthod. Dentofacial Orthop., St. Louis, v. 110, no. 4, p. 417-422, 1996.

28. VIGORITO, J. W. Ortodontia clínica: diagnóstico e terapêuticas. 1. ed. São Paulo: Santa Madonna, 2004. p. 49-98.

29. WIESLANDER, L. Intensive treatment of severe Class II malocclusions with a headgear-Herbst appliance in the early mixed dentition. Am. J. Orthod., St. Louis, v. 86, no. 1, p. 1-13, 1984

30. WILLIAMS, S.; MELSEN, B. The interplay between sagittal and vertical growth factors: An implant study of activator treatment. Am. J. Orthod., St. Louis, v. 81, no. 4, p. 327-332, 1982.
Endereço para correspondência

Luís Antônio de Arruda Aidar

Rua Luís Suplicy 35, Gonzaga

CEP: 11.055-330 - Santos / SP

E-mail: luisaidar@uol.com.br 